\title{
ORIGINAL
}

\section{PÉRDIDAS LABORALES OCASIONADAS POR MUERTES PREMATURAS EN ESPAÑA: UN ANÁLISIS PARA EL PERIODO 2005-2009 (*)}

\author{
Luz María Peña Longobardo (1), Isaac Aranda-Reneo (2), Juan Oliva Moreno (1) y \\ Judit Vall Castello (3).
}

(1) Facultad de Ciencias Jurídicas y Sociales. Toledo. Universidad de Castilla La Mancha.

(2) Facultad de Ciencias Sociales. Talavera de la Reina. Universidad de Castilla La Mancha.

(3) Facultad de Económicas. Universidad de Girona. Centro de Investigación de Economía y Salud. Universidad Pompeu Fabra.

(*) Este trabajo ha recibido una ayuda del Instituto de Estudios Fiscales para su elaboración. Asimismo, también se beneficia del apoyo del proyecto ECO2013-48217-C2-2-R "Impacto económico, sanitario y social de las enfermedades y los problemas de salud: información y herramientas para la evaluación de políticas públicas".

Los autores declaran no tener conflictos de intereses.

\section{RESUMEN}

Fundamentos: El impacto económico que causan las enfermedades va mucho más allá del gasto sanitario, transmitiéndose a la sociedad a través de diferentes dimensiones. El principal objetivo de este estudio fue estimar la evolución de las pérdidas de productividad laboral causadas por muertes prematuras en España durante el periodo 2005-2009.

Métodos: Se emplearon varias fuentes estadísticas (Registro de defunciones según la causa de muerte, Encuesta de Población Activa y Encuesta de Estructura Salarial) para desarrollar un modelo de simulación basado en el enfoque de capital humano que permitió estimar las pérdidas laborales ocasionadas por muertes prematuras para el periodo analizado. Adicionalmente, se llevaron a cabo dos escenarios alternativos en los que se analizó la influencia de la evolución epidemiológica en los resultados obtenidos.

Resultados: El modelo de simulación mostró una caída de las pérdidas laborales asociadas a muertes prematuras que fue desde 8.935 millones de euros en 2005 a 8.073 millones de euros en 2009. El análisis de las causas reducidas de muerte señaló que nueve causas de enfermedad (accidentes de tráfico, tumor maligno de tráquea, suicidios, infarto agudo de miocardio, SIDA, cirrosis, tumor de mama, enfermedades cerebrovasculares y cáncer de colon) supusieron el 35,6\% del total de Años Potenciales de Vida Laboral Perdidos y $36,6 \%$ del total de pérdida estimada. Las pérdidas estimadas representaron el $0,98 \%, 0,85 \%$ y $0,77 \%$ del Producto Interior Bruto de 2005 , 2007 y 2009 , respectivamente.

Conclusiones: Las pérdidas laborales ocasionadas por muertes prematuras en España disminuyeron sustancialmente en el periodo analizado. Esta reducción se debió fundamentalmente a la favorable evolución epidemiológica de la mortalidad prematura.

Palabras clave: Economía de la Salud, Economía, Análisis de Costes, Eficiencia, Mortalidad prematura.

Correspondencia

Luz María Peña Longobardo

Universidad de Castilla la Mancha

Facultad de Ciencias Jurídicas y Sociales de Toledo

Análisis Económico y Finanzas

Cobertizo de San Pedro Mártir s/n

45071 Toledo

LuzMaria.Pena@uclm.es

\section{ABSTRACT}

\section{Change in Productivity Losses Due to Premature Deaths in Spain: 2005-2009}

Background: The economic impact caused by diseases goes far beyond health care costs and, therefore it is transferred to the society through different dimensions. The aim of this study was to estimate the productivity losses due to premature deaths caused by diseases occurred in Spain during the period 2005-2009.

Methods: We used data from several sources (Death Registry, Labour Force Survey and Wage Structure Survey) to develop a simulation model based on the human-capital approach that allowed us to estimate the labour productivity losses caused by premature deaths in the period analysed. Additionally, we also carried out two alternative scenarios in which we analysed how epidemiologic data influenced our results.

Results: Our model showed the estimated loss of productivity due to premature death fell from 8,935 billion euros in 2005 to 8,073 billion euros in 2009. Nine diseases (traffic accidents, malignant tumour of the trachea, suicides, acute myocardial infarction, AIDS, cirrhosis, breast tumour, cerebrovascular disease and colon cancer) accounted for $35.6 \%$ of the total Years of Potential Productive Life Lost and $36.5 \%$ of the estimated productivity losses. The estimated losses represented $0.98 \%, 0.85 \%$ and $0.77 \%$ of Gross Domestic Product in 2005, 2007 and 2009, respectively.

Conclusions: The labour productivity losses caused by premature deaths decreased substantially in the period analysed. This reduction was mainly due to the epidemiological evolution of premature mortality.

Keywords: Health Economics, Economics, Cost and Cost Analysis, Efficiency, Mortality premature. 


\section{INTRODUCCIÓN}

La salud es uno de los pilares donde se apoya el bienestar de los individuos y las sociedades ${ }^{1}$. Asimismo, desde la óptica económica, la salud es un bien de naturaleza dual. Además de ser valorada por sí misma, que una sociedad disfrute de un buen estado de salud no garantiza el desarrollo económico, pero sí es un requisito esencial para ello ${ }^{2,3}$. Por consiguiente, la salud podría considerarse como una dimensión fundamental del capital humano en el que las inversiones actuales se utilizan para mejorarla, lo cual, a su vez, puede lograr un mayor desarrollo económico futuro ${ }^{4}$.

Cuando el impacto social que provocan determinadas enfermedades es evaluado desde una perspectiva económica, se debe considerar no solo el volumen de recursos invertidos en la atención sanitaria sino también determinados indicadores relacionados directamente con el impacto sobre el bienestar social que enfermedades y lesiones provocan (por ejemplo mortalidad, morbilidad, discapacidad, pérdidas en calidad de vida tanto en las pacientes como en el entorno afectivo, etcétera). En este sentido, en la literatura científica hay una tendencia creciente de revelar la carga económica que ocasionan determinadas enfermedades incorporando la perspectiva social en los análisis de coste de la enfermedad, lo cual supone ir más allá de los recursos estrictamente sanitarios empleados, modificados o perdidos a consecuencia de las enfermedades y lesiones ${ }^{5-8}$. Sin embargo, a pesar de identificar la importancia que pueden llegar a tener dichos costes no sanitarios, aún existen importantes lagunas en el caso de las pérdidas laborales que enfermedades y problemas de salud ocasionan. Aunque existen trabajos recientes que han estudiado el impacto ocasionado por la mortalidad prematura desde una óptica epidemiológica, ${ }^{9,10}$, así como estudios centrados en el impacto económico que provocan determinadas enfermedades ${ }^{11-13}, y$ estudios de coste de la enfermedad que incorporan las pérdidas laborales por muerte prematura como parte de sus estimaciones ${ }^{8,14-16}$, no existen apenas trabajos que analicen el impacto económico de las pérdidas laborales que ocasionan todas las enfermedad y lesiones en un país. Mucho menos aún estudios que analicen su evolución durante un periodo de 5 años.

El objetivo de este trabajo fue estimar las pérdidas de productividad ocasionadas por las muertes prematuras ocurridas en España durante el periodo 2005-2009. Adicionalmente, se analizó la influencia de la evolución epidemiológica sobre las pérdidas estimadas a lo largo del periodo.

\section{MATERIAL Y MÉTODOS}

Se partió de un trabajo previo que tomó como referencia el año $2005^{17,18}$. Dada la dificultad de observar y medir de manera directa la productividad laboral de cada persona, la teoría económica considera que el salario bruto obtenido es una variable razonable para medir la productividad ${ }^{19-21}$. Así, según las teorías del capital humano ${ }^{21,22}$, la ganancia salarial de un trabajador se puede utilizar como base para la estimación de la corriente de la productividad laboral perdida cuando deja el mercado laboral a consecuencia de una enfermedad o lesión.

Se utilizaron las Encuestas de Estructura Salarial (EES) de los años 2005, 2007 y 2009 del Instituto Nacional de Estadística para obtener el salario bruto medio según el sexo, la edad y la Comunidad Autónoma de residencia ${ }^{23-25}$. Los microdatos del Registro de Defunciones Según la Causa de Muerte proporcionaron la información sobre los fallecimientos acaecidos dentro del territorio nacional atendiendo a la causa básica que los determinó, el sexo, la edad y la Comunidad Autónoma de residencia ${ }^{26-28}$. Este registro emplea la Clasificación Internacional de Enfermedades de la Organización Mundial de la Salud $\left(10^{a}\right.$ revisión de dicha clasificación $^{29}$. Al contar con los microdatos del Registro de defunciones fue posible excluir 
de las estimaciones los fallecimientos de personas que no tenían su residencia en España, ganando así una mayor precisión en la estimación con respecto a trabajos previos $^{17,18}$. Por su parte, la Encuesta de Población Activa se empleó para obtener la tasa de ocupación según el sexo, la edad y la Comunidad Autónoma de residencia para cada uno de los años en los que se estimaron las pérdidas de productividad (años 2005, 2007 y 2009) ${ }^{30-32}$. Dicha tasa fue definida como el porcentaje de la población ocupada en relación con la población en edad de trabajar.

De este modo, con la información proporcionada por el Registro de Defunciones, los salarios y la tasa de ocupación, se realizó el cálculo del flujo presente y futuro de la productividad laboral perdida por una muerte prematura ocasionada por cualquiera de las causas consideradas. Con este fin, para cada muerte producida en un grupo de edad y sexo determinado, se aplicó la tasa de ocupación y ganancia salarial esperados en cada periodo posterior hasta el límite prefijado (en nuestro caso, los 65 años por ser la edad de jubilación en España durante el periodo). Los fallecimientos producidos en menores de 16 años (fuera del mercado laboral) se tuvieron en cuenta dentro de las estimaciones, ya que en el futuro, si estas personas no hubieran fallecido prematuramente, se habrían incorporado al mercado laboral a partir de la edad legal establecida. Las probabilidades de empleo para cada edad fueron ajustadas a través de las tasas de ocupación observadas en cada año considerado. A los valores futuros obtenidos se les aplicó una tasa anual de descuento del tres por ciento y una tasa anual de crecimiento de la productividad laboral del uno por ciento. Este fue considerado el caso base sobre el cual se realizó un análisis de sensibilidad considerando dos tasas de descuento alternativas, cero por ciento y seis por ciento, y dos tasas de crecimiento de la productividad laboral, cero por ciento y dos por ciento. Además de la estimación de las pérdidas laborales, también se estimaron los
Años Potenciales de Vida Laboral Perdidos (APVLP). En el caso de fallecimientos de personas menores de 16 años, los APVLP de cada muerte se estimaron como la diferencia entre los 65 años y la edad legal para iniciar la etapa laboral (16 años).

Para analizar si la evolución de las pérdidas de productividad se debía a la tendencia seguida en el mercado laboral (salarios y tasas de empleo) o a la tendencia de los datos epidemiológicos (número de fallecimientos y APVLP) se diseñaron dos escenarios alternativos. La estimación para el año 2009 fue reevaluada considerando los cambios epidemiológicos (esto es, distribución de fallecimientos por edad y sexo) de los años 2005 y 2007 . Mediante este ejercicio, aislamos el efecto de los cambios epidemiológicos sobre otros efectos del mercado laboral (salarios y tasa de empleo) que pueden influir en el análisis.

\section{RESULTADOS}

El número de fallecimientos totales en España fue de 385.056 en el año 2005, 383.249 en 2007 y 383.209 en 2009 (tabla 1). La distribución del número de muertes durante todo el periodo fue del $52 \%$ de los varones mientras que el $48 \%$ restante fueron mujeres). El número total de muertes disminuyó durante 2005 y 2007, mientras que se mantuvo entre 2007 y 2009. En los cinco años considerados, el número de fallecimientos disminuyó de manera generalizada, excepto en cuatro grupos de enfermedades: tumores, enfermedades del sistema nervioso, enfermedades de la piel y enfermedades del sistema genitourinario. El número de fallecimientos en personas menores de 65 años descendió en $2,7 \%$ entre los años 2005 y 2009, mostrando un total de 1.744 muertes menos. El mayor número de muertes en menores de 65 años se produjo por enfermedades del sistema circulatorio, tumores y causas externas de mortalidad.

En lo que se refiere a los APVLP, disminuyeron en un $9,8 \%$ durante el periodo 


\begin{tabular}{|c|c|c|c|c|c|c|c|c|c|}
\hline \multicolumn{10}{|c|}{$\begin{array}{c}\text { Tabla } 1 \\
\text { Número de fallecimientos en España, Años Potenciales de Vida Laboral Perdidos (APVLP) según causa }\end{array}$} \\
\hline & \multicolumn{3}{|c|}{ Fallecimientos(a) } & \multicolumn{3}{|c|}{$\begin{array}{l}\text { Fallecimientos } \\
\text { en edad laboral } \\
(<65 \text { años })\end{array}$} & \multicolumn{3}{|c|}{ APVLP } \\
\hline & 2005 & 2007 & 2009 & 2005 & 2007 & 2009 & 2005 & 2007 & 2009 \\
\hline Enfermedades infecciosas y parasitarias & 7.452 & 7.737 & 6.813 & 2.506 & 2.369 & 1.984 & 50.863 & 46.604 & 36.685 \\
\hline Tumores & 99.949 & 103.088 & 104.918 & 26.234 & 27.112 & 27.241 & 300.316 & 301.959 & 298.753 \\
\hline Enf. de sangre y de órg. hematopoyéticos & 1.311 & 1.277 & 1.456 & 185 & 192 & 194 & 4.035 & 4.224 & 3.889 \\
\hline Enfermedades endocrinas, nutricionales y metabólicas & 12.389 & 12.206 & 12.417 & 1.146 & 1.148 & 1.151 & 15.425 & 15.421 & 15.919 \\
\hline Trastornos mentales y del comportamiento & 12.526 & 12.304 & 14.451 & 472 & 373 & 440 & 8.117 & 5.074 & 5.585 \\
\hline Enfermedades del sistema nervioso y de los órganos de los sentidos & 15.665 & 16.699 & 18.748 & 1.664 & 1.680 & 1.673 & 33.532 & 31.682 & 31.197 \\
\hline Enfermedades del sistema circulatorio & 125.933 & 123.250 & 119.366 & 11.374 & 11.329 & 10.775 & 131.888 & 130.276 & 123.150 \\
\hline Enfermedades del sistema respiratorio & 46.906 & 43.899 & 43.178 & 3.028 & 3.134 & 3.077 & 40.642 & 42.445 & 42.620 \\
\hline Enfermedades del sistema digestivo & 19.515 & 19.543 & 19.460 & 3.959 & 4.147 & 3.938 & 49.940 & 52.256 & 47.925 \\
\hline Enfermedades de la piel y del tejido subcutáneo & 1.105 & 1.184 & 1.194 & 49 & 49 & 56 & 622 & 637 & 694 \\
\hline Enfermedades del sistema osteomuscular y del tejido conjuntivo & 3.584 & 3.376 & 3.451 & 224 & 182 & 204 & 3.499 & 2.353 & 2.813 \\
\hline Enfermedades del sistema genitourinario & 9.534 & 10.141 & 10.838 & 510 & 517 & 488 & 5.937 & 5.566 & 5.547 \\
\hline Embarazo, parto y puerperio & 18 & 13 & 17 & 18 & 13 & 17 & 574 & 394 & 556 \\
\hline Afecciones originadas en el periodo perinatal & 924 & 961 & 884 & 923 & 959 & 882 & 44.970 & 46.753 & 43.040 \\
\hline Malformaciones congénitas, deformidades y anomalías cromosómicas & 950 & 880 & 875 & 858 & 794 & 777 & 34.602 & 30.915 & 29.815 \\
\hline Síntomas, signos y hallazgos anormales clínicos y de laboratorio, NCOP & 10.844 & 11.247 & 10.966 & 1.829 & 2.128 & 2.352 & 36.408 & 41.088 & 44.826 \\
\hline Causas externas de mortalidad & 16.451 & 15.444 & 14.177 & 9.323 & 8.552 & 7.309 & 246.428 & 217.656 & 175.988 \\
\hline Todas las causas & 385.056 & 383.249 & 383.209 & 64.302 & 64.678 & 62.558 & 1.007 .798 & 975.303 & 909.002 \\
\hline
\end{tabular}




\section{Figura 1}

\section{Evolución de los Años Potenciales de Vida Laboral Perdidos (APVLP) durante 2005-2007-2009}

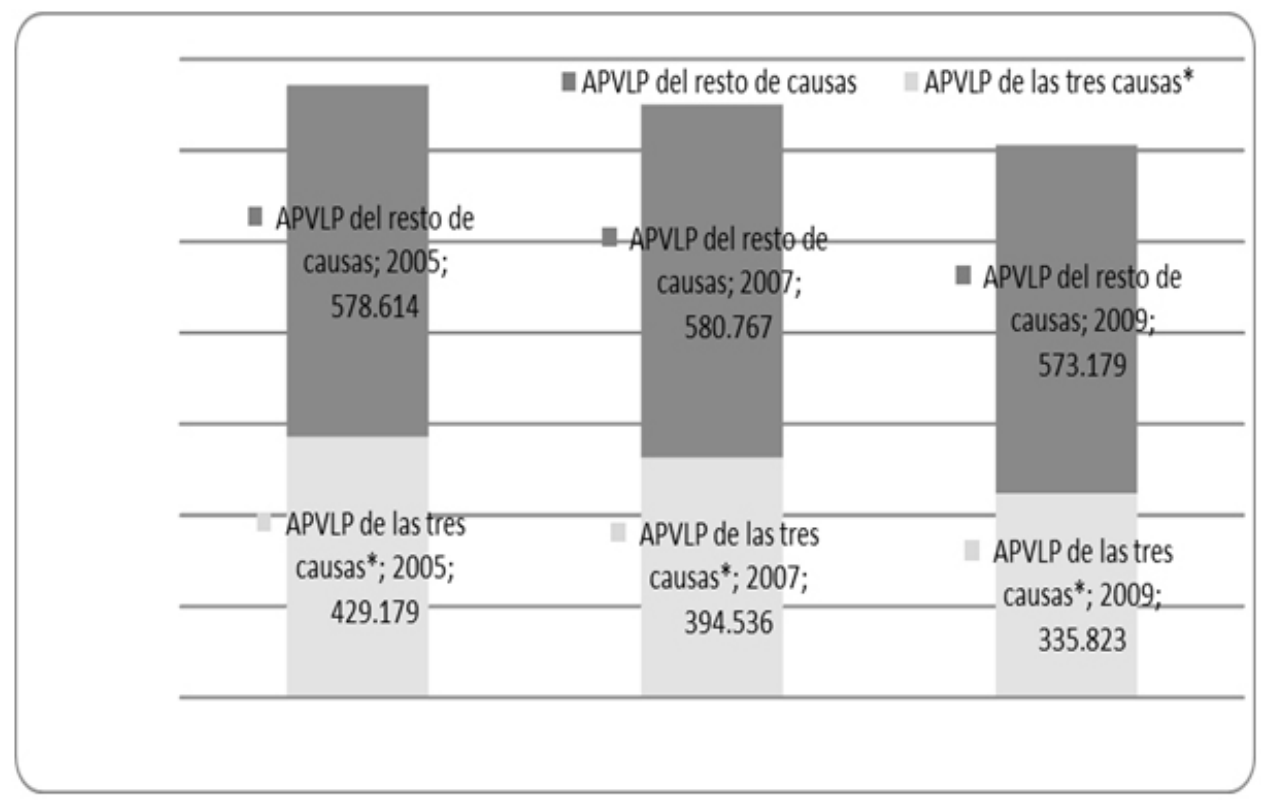

*Nota: Grupo II: Tumores; Grupo IX: Enfermedades del sistema circulatorio; Grupo XX: Causas externas de mortalidad.

Fuente: Elaboración propia a partir de distintas fuentes

analizado pasando de 1.007.798 APVLP en 2005 a 909.002 APVLP en 2009. Al contrario de lo que ocurrió con el número de fallecimientos, los APVLP mostraron una disminución mucho más marcada durante el periodo 2007-2009 que durante el periodo 2005-2007 (66.310 APVLP menos durante 2007-2009 comparado con 32.492 APVLP menos durante 2005-2007). La caída en el número de APVLP fue más evidente que el número de fallecimientos $(9,8 \%$ comparado con $0,5 \%$ ). Ello fue resultado del efecto simultáneo de una disminución en el número de fallecimientos prematuros $\mathrm{y}$ del incremento de la edad media a la que acontecieron estos fallecimientos prematuros. En términos generales, los APVLP por grupo de enfermedad fueron menores en la mayoría de las categorías estudiadas. Entre los grupos de enfermedades que más contribuyeron a la reducción global de los
APVLP se encontraron las causas externas de mortalidad, enfermedades infeccionas y parasitarias y las enfermedades del sistema respiratorio. Durante los años analizados la causa de enfermedad con la cifra más elevada de APVLP fueron los tumores, seguidos de las causas externas de mortalidad y las enfermedades del sistema circulatorio, representando un 33\%, 13\% y 19\% sobre el total de APVLP, respectivamente (figura 1).

La tabla 2 recoge la estimación de las perdidas laborales ocasionadas por la mortalidad prematura asociadas a diferentes grupos de enfermedad durante el periodo considerado. En el escenario base la pérdida laboral por muertes prematuras fue de 8.935,4 millones de euros en el año 2005, $8.937,3$ millones en el año 2007 y 8.073,5 millones en el año 2009 (valores nominales). Las causas de muerte que dieron lugar a 
Tabla 2

Pérdidas laborales por causa de enfermedad. Millones de euros

\begin{tabular}{|c|c|c|c|c|c|c|c|c|c|}
\hline & 2005 & 2007 & 2009 & $\begin{array}{c}2009^{*} \\
\text { Escenario } 1\end{array}$ & $\begin{array}{c}\text { Diferencia } \\
\text { absoluta }\end{array}$ & $\begin{array}{c}\text { Diferencia } \\
\text { relativa } \\
\%\end{array}$ & $\begin{array}{c}2009^{*} \\
\text { Escenario } 2\end{array}$ & $\begin{array}{c}\text { Diferencia } \\
\text { absoluta }\end{array}$ & $\begin{array}{c}\text { Diferencia } \\
\text { relativa } \\
\%\end{array}$ \\
\hline Enfermedades infecciosas y parasitarias & 522,72 & 493,35 & 365,73 & 530,18 & 164,45 & $44,96 \%$ & 482,89 & 117,16 & $32,03 \%$ \\
\hline Tumores & $2.372,23$ & $2.477,67$ & $2.422,52$ & $2.482,95$ & 60,43 & $2,49 \%$ & $2.483,11$ & 60,59 & $2,50 \%$ \\
\hline Enf. de sangre y de órg. hematopoyéticos & 34,65 & 37,08 & 33,38 & 35,44 & 2,06 & $6,17 \%$ & 36,53 & 3,15 & $9,44 \%$ \\
\hline Enfermedades endocrinas, nutricionales y metabólicas & 125,45 & 130,46 & 134,05 & 122,76 & $-11,29$ & $-8,42 \%$ & 128,80 & $-5,25$ & $-3,92 \%$ \\
\hline Trastornos mentales y del comportamiento & 82,30 & 50,54 & 52,39 & 83,12 & 30,73 & $58,66 \%$ & 49,54 & $-2,85$ & $-5,44 \%$ \\
\hline Enfermedades del sistema nervioso y de los órganos de los sentidos & 278,16 & 276,69 & 261,59 & 288,23 & 26,64 & $10,18 \%$ & 272,26 & 10,67 & $4,08 \%$ \\
\hline Enfermedades del sistema circulatorio & $1.187,59$ & $1.215,50$ & $1.131,38$ & $1.204,36$ & 72,98 & $6,45 \%$ & $1,196,83$ & 65,45 & $5,78 \%$ \\
\hline Enfermedades del sistema respiratorio & 364,02 & 395,45 & 383,92 & 371,30 & $-12,62$ & $-3,29 \%$ & 388,99 & 5,07 & $1,32 \%$ \\
\hline Enfermedades del sistema digestivo & 472,29 & 508,34 & 451,79 & 476,88 & 25,09 & $5,55 \%$ & 498,42 & 46,63 & $10,32 \%$ \\
\hline Enfermedades de la piel y del tejido subcutáneo & 4,95 & 4,85 & 5,75 & 5,10 & $-0,65$ & $-11,30 \%$ & 4,96 & $-0,79$ & $-13,74 \%$ \\
\hline Enfermedades del sistema osteomuscular y del tejido conjuntivo & 26,39 & 17,57 & 21,96 & 28,73 & 6,77 & $30,83 \%$ & 18,08 & $-3,88$ & $-17,67 \%$ \\
\hline Enfermedades del sistema genitourinario & 46,40 & 45,29 & 46,51 & 48,87 & 2,36 & $5,07 \%$ & 45,60 & $-0,91$ & $-1,96 \%$ \\
\hline Embarazo, parto y puerperio & 3,37 & 2,88 & 4,28 & 4,26 & $-0,02$ & $-0,47 \%$ & 3,16 & -1.12 & $-26,17 \%$ \\
\hline Afecciones originadas en el periodo perinatal & 329,21 & 354,60 & 319,25 & 335,00 & 15,75 & $4,93 \%$ & 342,35 & 23,1 & $7,24 \%$ \\
\hline Malformaciones congénitas, deformidades y anomalías cromosómicas & 255,83 & 243,84 & 227,46 & 263,28 & 35,82 & $15,75 \%$ & 236,91 & 9,45 & $4,15 \%$ \\
\hline Síntomas, signos y hallazgos anormales clínicos y de laboratorio, NCOP & 340,86 & 406,00 & 429,15 & 345,58 & $-83,57$ & $-19,47 \%$ & 395,77 & $-33,38$ & $-7,78 \%$ \\
\hline Causas externas de mortalidad & $2.488,88$ & $2.277,10$ & $1.782,27$ & $2.500,76$ & 718,49 & $40,31 \%$ & $2.202,94$ & 420,67 & $23,60 \%$ \\
\hline Todas las causas & $8.935,40$ & $8.937,29$ & $8.073,46$ & $9.133,59$ & $1.060,13$ & $13,13 \%$ & $8.787,22$ & 713,76 & $8,84 \%$ \\
\hline
\end{tabular}

*Pérdidas de productividad estimadas en 2009 a partir de datos epidemiológicos de 2005 (b) Pérdidas de productividad estimadas en 2009 a partir de datos epidemiológicos de 2007. 
las mayores pérdidas de productividad en el período analizado fueron los tumores (que representaron el $29,30 \%$ del total de pérdidas en $2005,29,44 \%$ en 2007 y $30,03 \%$ en 2009), las causas externas de mortalidad (que representaron 22,49\% de las pérdidas totales en $2005,22,46 \%$ en 2007 y $22,08 \%$ en 2009), y las enfermedades del sistema circulatorio.

Si en el año 2009 se hubiera producido la misma pauta de fallecimientos que en el año 2005, las pérdidas estimadas habrían ascendido de 8.073 a 9.134 millones de euros. Esto representa una diferencia de más de 1.061 millones de euros (un 13,13\% con respecto al escenario base). Aplicando el mismo procedimiento al año 2007 , si en el año 2009 se hubiera producido la misma pauta de fallecimiento que en el año 2007, las pérdidas estimadas habrían ascendido de 8.073 a 8.787 millones de euros. Es decir, un aumento de 714 millones de euros en la pérdida de la productividad total. Los resultados obtenidos en términos de APVLP muestran una evolución favorable a lo largo del periodo, coincidente con los resultados de las pérdidas laborales ya señalados.

Si tomáramos en consideración una tasa de descuento del $6 \%$ y una tasa de crecimiento de productividad del $0 \%$, las pérdidas laborales se estimarían entre 6.509,98 millones en 2005 y 5.974,26 en 2009 (tabla 3). Si por el contrario tomáramos en cuenta el escenario opuesto, una tasa de descuento

Tabla 3

Pérdidas laborales por causa de enfermedad. Análisis de sensibilidad con tasas de descuento alternativas. Millones de euros

\begin{tabular}{|c|c|c|c|c|c|c|}
\hline & \multicolumn{2}{|c|}{2005} & \multicolumn{2}{|c|}{2007} & \multicolumn{2}{|c|}{2009} \\
\hline & $6 \%-0 \%{ }^{*}$ & $0 \%-2 \% \dagger$ & $6 \%-0 \%{ }^{*}$ & $0 \%-2 \%{ }^{\dagger}$ & $6 \%-0 \%{ }^{*}$ & $0 \%-2 \% \dagger$ \\
\hline Enfermedades infecciosas y parasitarias & 387,28 & 810,71 & 366,10 & 753,98 & 275,2 & 550,84 \\
\hline Tumores & $1.896,00$ & $3.306,73$ & $1.983,24$ & $3.436,38$ & $1.940,89$ & $3.353,8$ \\
\hline Enf. de sangre y de órg. hematopoyéticos & 22,47 & 66,42 & 24,53 & 70,75 & 22,16 & 62,07 \\
\hline Enfermedades endocrinas, nutricionales y metabólicas & 90,08 & 212,28 & 93,90 & 219,52 & 96,82 & 223,32 \\
\hline Trastornos mentales y del comportamiento & 62,88 & 126,61 & 39,28 & 73,71 & 40,86 & 75,95 \\
\hline Enfermedades del sistema nervioso y de los órganos de los sentidos & 184,75 & 522,75 & 185,18 & 512,56 & 175,65 & 484,14 \\
\hline Enfermedades del sistema circulatorio & 945,79 & $1.666,04$ & 965,76 & $1.705,62$ & 899,48 & $1.586,20$ \\
\hline Enfermedades del sistema respiratorio & 274,22 & 569,08 & 298,30 & 616,21 & 288,97 & 599,09 \\
\hline Enfermedades del sistema digestivo & 377,84 & 643,69 & 405,01 & 699,80 & 364,05 & 606,86 \\
\hline Enfermedades de la piel y del tejido subcutáneo & 4,09 & 6,72 & $3.706,31$ & 7,43 & 4,41 & 8,54 \\
\hline Enfermedades del sistema osteomuscular y del tejido conjuntivo & 19,51 & 41,42 & 13,489 & 26,28 & 17,01 & 31,62 \\
\hline Enfermedades del sistema genitourinario & 36,262 & 67,41 & 35,98 & 63,19 & 36,55 & 66,21 \\
\hline Embarazo, parto y puerperio & 2,26 & 5,63 & 1,97 & 4,64 & 2,85 & 7,20 \\
\hline Afecciones originadas en el periodo perinatal & 151,64 & 862,13 & 164,78 & 930,02 & 143,66 & 857,12 \\
\hline Malformaciones congénitas, deformidades y anomalías cromosómicas & 129,03 & 628,63 & 126,41 & 590,20 & 117,72 & 553,10 \\
\hline Síntomas, signos y hallazgos anormales clínicos y de laboratorio, NCOP & 241,35 & 585,61 & 291,52 & 681,50 & 306,51 & 725,30 \\
\hline Causas externas de mortalidad & $1.684,45$ & $4.633,01$ & $1.566,94$ & $4.131,57$ & $1.241,48$ & $3.174,50$ \\
\hline Todas las causas & $6.509,98$ & $14.754,94$ & $6.566,18$ & $14.523,43$ & $5.974,26$ & $12.965,85$ \\
\hline
\end{tabular}

* Tasa de descuento $6 \%$ y tasas crecimiento productividad $0 \%$. ${ }^{\dagger}$ Tasa de descuento $0 \%$ y tasas crecimiento productividad $2 \%$.

Nota: los resultados obtenidos en los escenarios de valoración restantes están a disposición de los lectores tras petición a los autores 
Tabla 4

Pérdidas laborales y Años Potenciales de Vida Laboral Perdidos (APVLP) causados por 9 enfermedades y lesiones

\begin{tabular}{|c|c|c|c|c|c|c|}
\hline & \multicolumn{3}{|c|}{$\begin{array}{l}\text { APVLP } \\
\text { Total } \\
\left(\%{ }^{*}\right)\end{array}$} & \multicolumn{3}{|c|}{$\begin{array}{c}\text { Pérdidas laborales } \\
\text { (en millones de euros) } \\
\text { Total } \\
\left(\%{ }^{*}\right)\end{array}$} \\
\hline & 2005 & 2007 & 2009 & 2005 & 2007 & 2009 \\
\hline Accidentes de tráfico & $\begin{array}{r}102.699 \\
(9,98)\end{array}$ & $\begin{array}{r}8.478 \\
(8,48)\end{array}$ & $\begin{array}{r}53.351 \\
(5,87)\end{array}$ & $\begin{array}{r}1.024,59 \\
(11,21)\end{array}$ & $\begin{array}{r}882,51 \\
(9,64)\end{array}$ & $\begin{array}{r}535,54 \\
(6,63)\end{array}$ \\
\hline Tumor maligno de la tráquea, de los bronquios y del pulmón & $\begin{array}{r}61.816 \\
(6,00)\end{array}$ & $\begin{array}{r}64.728 \\
(6,49)\end{array}$ & $\begin{array}{r}62.421 \\
(6,87)\end{array}$ & $\begin{array}{r}542,24 \\
(5,94)\end{array}$ & $\begin{array}{r}572,34 \\
(6,25)\end{array}$ & $\begin{array}{r}489,98 \\
(5,35)\end{array}$ \\
\hline Suicidios y lesiones autoinfligidas & $\begin{array}{r}51.646 \\
(5,02)\end{array}$ & $\begin{array}{r}46.986 \\
(4,71)\end{array}$ & $\begin{array}{r}50.507 \\
(5,56)\end{array}$ & $\begin{array}{r}522,17 \\
(5,72)\end{array}$ & $\begin{array}{r}489,98 \\
(5,35)\end{array}$ & $\begin{array}{r}515,43 \\
(6,38\end{array}$ \\
\hline Infarto agudo de miocardio & $\begin{array}{r}39.151 \\
(3,80)\end{array}$ & $\begin{array}{r}36.030 \\
(3,61)\end{array}$ & $\begin{array}{r}33.939 \\
(3,73)\end{array}$ & $\begin{array}{r}372,44 \\
(4,08)\end{array}$ & $\begin{array}{r}348,43 \\
(3,81)\end{array}$ & $\begin{array}{r}326,44 \\
(4,04)\end{array}$ \\
\hline Sida & $\begin{array}{r}32.550 \\
(3,16)\end{array}$ & $\begin{array}{r}27.328 \\
(2,74)\end{array}$ & $\begin{array}{r}20.864 \\
(2,30)\end{array}$ & $\begin{array}{r}358,76 \\
(3,93)\end{array}$ & $\begin{array}{r}311,97 \\
(3,41) \\
\end{array}$ & $\begin{array}{r}224,36 \\
(2,78)\end{array}$ \\
\hline Cirrosis y otras enfermedades del hígado & $\begin{array}{r}31.210 \\
(3,03)\end{array}$ & $\begin{array}{r}31.823 \\
(3,19)\end{array}$ & $\begin{array}{r}29.827 \\
(3,28)\end{array}$ & $\begin{array}{r}301,95 \\
(3,30)\end{array}$ & $\begin{array}{r}316,14 \\
(3,45)\end{array}$ & $\begin{array}{r}285,18 \\
(3,53)\end{array}$ \\
\hline Tumor maligno de la mama & $\begin{array}{r}28.294 \\
(2,75)\end{array}$ & $\begin{array}{r}30.157 \\
(3,02)\end{array}$ & $\begin{array}{r}29.435 \\
(3,24)\end{array}$ & $\begin{array}{r}131,81 \\
(1,44)\end{array}$ & $\begin{array}{r}163,64 \\
(1,79)\end{array}$ & $\begin{array}{r}177,61 \\
(2,20)\end{array}$ \\
\hline Enfermedades cerebrovasculares & $\begin{array}{r}28.342 \\
(2,75)\end{array}$ & $\begin{array}{r}27.895 \\
(2,80)\end{array}$ & $\begin{array}{r}25.339 \\
(2,79)\end{array}$ & $\begin{array}{r}234,28 \\
(2,56)\end{array}$ & $\begin{array}{r}242,23 \\
(2,65)\end{array}$ & $\begin{array}{r}219,35 \\
(2,72)\end{array}$ \\
\hline Cáncer de colon & $\begin{array}{r}17.094 \\
(1,66)\end{array}$ & $\begin{array}{r}17.655 \\
(1,77)\end{array}$ & $\begin{array}{r}18.164 \\
(2,00)\end{array}$ & $\begin{array}{r}127,98 \\
(1,40)\end{array}$ & $\begin{array}{r}140,64 \\
(1,54)\end{array}$ & $\begin{array}{r}140,83 \\
(1,74)\end{array}$ \\
\hline Las 9 causas seleccionadas & 392.802 & 367.180 & 323.847 & $3.616,25$ & $3.467,86$ & $2.954,46$ \\
\hline Todas las causas & 1.007 .793 & 975.903 & 909.002 & $8.935,40$ & $8.937,20$ & $8.073,46$ \\
\hline$\%$ de las 9 enfermedades y lesiones mencionadas sobre el total & 38,97 & 37,62 & 35,63 & 40,47 & 38,80 & 36,59 \\
\hline
\end{tabular}

* Porcentaje sobre todas las causas de mortalidad.

del $0 \%$ y de productividad el $2 \%$, la pérdida estimada se situaría en 14.754,94 millones para 2005 y en $12.965,85$ en 2009 .

En la tabla 4 se muestran los resultados de las causas de enfermedad con mayor impacto por su mortalidad prematura asociada. En concreto, nueve causas de enfermedad (por causa reducida) representaron entre el 36\%$40 \%$, dependiendo del año, del total de pérdidas y entre 35\%-39\% del total de APVLP en el período analizado. Los accidentes de tráfico provocaron el mayor nivel de pérdidas de productividad laboral y de APVLP, seguidos de los tumores de tráquea, bronquios y pulmón así como los suicidios. En general, la tendencia observada en pérdidas de productividad y en los APVLP para estas nueve enfermedades fue positiva (menores pérdidas de productividad laboral y menos APVLP).
El tumor maligno de mama y el cáncer de colon fueron las dos únicas enfermedades de este grupo de nueve que mostraron un aumento en los APVLP y en las pérdidas de laborales estimadas.

\section{DISCUSIÓN}

El principal resultado de este estudio muestra una disminución de las pérdidas laborales causadas por muertes prematuras en España, aun en términos nominales, durante el período comprendido entre los años 2005 y 2009. Las pérdidas estimadas representan el $0,98 \%, 0,85 \%$ y $0,77 \%$ del Producto Interior Bruto (PIB) de 2005, 2007 y 2009, respectivamente. Esta comparación entre el PIB y las pérdidas de productividad ayuda a revelar el potencial impacto de las muertes prematuras en la dimensión económica. Sin embargo, 
no se pretende sugerir que el PIB de España habría sido $0,98 \%, 0,85 \%$, y $0,77 \%$ superior en caso de no haberse producido ningún fallecimiento prematuro. La complejidad del mercado de trabajo tanto en su dimensión de cantidades (número de puestos de trabajo $\mathrm{y}$ las horas de trabajo) como en su dimensión de precios (salarios) y su traslación a otros aspectos económicos de producción, inversión y consumo previene de la proyección de micro-supuestos a consecuencias macroeconómicas. Sin embargo, dada la magnitud de las cifras de pérdidas estimadas, sí es útil tener un elemento de comparación de dichas cifras, siendo el PIB un indicador adecuado para ello.

Este trabajo presenta varios aspectos que no han sido analizados anteriormente en la literatura científica. En primer lugar, es el primero en examinar la evolución de las pérdidas laborales causadas por muertes prematuras (todas las causas) durante un período de cinco años. Además, hasta donde conocemos, es pionero en proponer un escenario donde los efectos del mercado laboral se aíslan del efecto puramente epidemiológico de la evolución en el número y tipo de fallecimiento (escenarios 1 y 2 de la tabla 2). De esta manera, se estimaron las pérdidas de productividad que se habrían producido en 2009 si el patrón de mortalidad no hubiera evolucionado favorablemente durante el periodo considerado. Por otro lado, la utilización de los microdatos del Registro de Defunciones permite mejorar las estimaciones realizadas por los autores en trabajos previos ${ }^{17,18}$. Aunque la diferencia en términos relativos es una reducción de apenas el 2,2\% respecto a la estimación original, el uso de los microdatos representa una mejora de la información que permite llevar a cabo estimaciones más precisas y favorecerá la comparación de los presentes resultados con las estimaciones de futuras investigaciones.

Comparando nuestros resultados con los de los trabajos previos realizados en Canadá y Estados Unidos en el año 2012 $2^{33,34}$, se pue- den apreciar pautas comunes. Por ejemplo, los tumores malignos, las causas externas y las enfermedades cardiovasculares presentan una gran relevancia sobre los fallecimientos prematuros y las pérdidas laborales. Sin embargo, fue extremadamente complicado comparar los resultados obtenidos con los de investigaciones más actuales debido a la escasez de trabajos en la literatura sobre la estimación de las pérdidas de la productividad laboral a nivel de país, ya que las publicaciones existentes se centran en el impacto económico de enfermedades concretas, no existiendo análisis del impacto económico total de las pérdidas laborales asociadas a todas las causas de muerte.

El enfoque teórico utilizado en el estudio es la teoría del capital humano. Cabe señalar que existen enfoques alternativos, tales como el método del período de fricción ${ }^{35}$. Algunos trabajos han discutido ampliamente las diferencias entre los dos métodos, sus fortalezas y debilidades ${ }^{36-38}$. Sin embargo, aunque la discusión metodológica de la idoneidad de un método frente a su alternativa está lejos de ser resuelto en la literatura ${ }^{39-41}$, la decisión de aplicar el enfoque de capital humano fue consensuado por los investigadores y el Instituto de Estudios Fiscales (IEF), ya que es el método más utilizado en la literatura científica de estudios de costes de enfermedad y además es coherente con el enfoque adoptado en otros estudios realizados o financiados por el IEF en distintas áreas de la hacienda pública y la economía aplicada.

En general, los resultados de este trabajo pueden ayudar a las autoridades públicas a avanzar en el diseño de sus políticas de salud. En este sentido, uno de los resultados más relevantes es la constatación de que tres grupos de enfermedades (causas externas de mortalidad, tumores y enfermedades del sistema circulatorio) son responsables de más del $50 \%$ de las pérdidas de productividad estimadas. Asimismo, se constata el gran impacto sobre la salud que tiene un reducido número de enfermedades y lesiones. La 
naturaleza de las mismas hace que, en una importante medida, los fallecimientos prematuros a consecuencia de dichas enfermedades y lesiones sean, si no eliminables por completo, sí susceptibles de reducción.

Con todo ello, nuestros resultados ponen de relieve una parte de las ventajas potenciales de la aplicación de estrategias de prevención primaria y/o secundaria en determinadas enfermedades en las que se concentran la mayor parte de las muertes prematuras y las pérdidas laborales en España. Asimismo, la implementación de programas, estrategias y políticas de promoción y cuidado de la salud efectivas no sólo tendrían ventajas directas sobre la salud de la población sino que también mejorarían su capital humano, lo que sería un factor añadido a tener en cuenta para la sostenibilidad de nuestro desarrollo económico futuro $\mathrm{y}$, considerando ambos aspectos, del bienestar de la población en el largo plazo.

Es evidente que la asignación de los recursos para la atención de la salud no debe basarse únicamente en el impacto de una enfermedad sino más bien en los potenciales beneficios para la salud de una determinada intervención o política, además de otras consideraciones como los problemas de equidad, las implicaciones éticas, las preferencias del población o los recursos disponibles, sin agotar la lista. No obstante, teniendo en cuenta sus limitaciones, la información sobre el impacto económico de las enfermedades y los estudios centrados en el retorno de la inversión de las intervenciones de salud ${ }^{4}$ sirven no para reemplazar sino para sumarse a la información epidemiológica y de otra naturaleza sobre los problemas de salud de la población ${ }^{42-46}$. Por lo tanto, los resultados del presente trabajo podrían servir para senalar aquellas áreas en las que políticas de salud pública podrían obtener potencialmente mayor impacto en la reducción de la carga de la enfermedad, así como en la mitigación del impacto económico ocasionado por las muertes prematuras.

\section{AGRADECIMIENTOS}

Los autores agradecen el apoyo institucional recibido del Instituto de Estudios Fiscales y el Instituto Nacional de la Seguridad Social (INSS) y dan las gracias a Angela Blanco y José María Labeaga (IEF) y María Dolores de Santa María y su equipo (INSS) por su ayuda y apoyo constante. También expresamos nuestro agradecimiento por los comentarios recibidos de Javier Hernández y los participantes del taller realizado en el Instituto de Estudios Fiscales, así como a los revisores que evaluaron el trabajo.

\section{BIBLIOGRAFÍA}

1. Graham C. Happiness and Health: Lessons-And Questions-For Public Policy. Health Aff. 2008; 27: 72-87.

2. Commission of the European Communities: White Paper-Together for Health: A Strategic Approach for the EU 2008-2013. 2007. (citado 2012). Disponible en: http://ec.europa.eu/health-eu/doc/whitepaper_en.pdf

3. Sachs JD. Macroeconomics and health: Investing in health for economic development. Report of the Commission on macroeconomics and health. World Health Organization. 2001. (citado 14/01/2013) Disponible en: http://whqlibdoc.who. int/publications/2001/924154550x.pdf

4. Suhrcke M, Mckee M, Sauto-Arce R, Tsolova S, Mortensen J. The contribution of health to the economy in the European Union. . Public Health. 2006; 120(11): 994-1001.

5. Ringborg A, Nieuwlaat R, Lindgren P, Jonsson B, Fidan D, Maggioni AP, et al. Costs of atrial fibrillation in five European countries: results from the Euro Heart Survey on atrial fibrillation. Europace. 2008 Apr; 10(4): 403-11.

6. Salize HJ, McCabe R, Bullenkamp J, Hansson L, Lauber C, Martinez-Leal R, et al. Cost of treatment of schizophrenia in six European countries. Schizophr Res. 2009; 111(1-3): 70-7.

7. Gustavsson A, Svensson M, Jacobi F, Allgulander C, Alonso J, Beghi E, et al. Cost of disorders of the brain in Europe 2010. Eur Neuropsychopharmacol. 2011 Oct; 21(10): 718-79.

8. Leal J, Luengo-Fernandez R, Gray A, Petersen S, Rayner M. Economic burden of cardiovascular diseases in the enlarged European Union. Eur Heart J. 2006 Jul; 27(13): 1610-9. 
9. Gènova-Maleras R, Catalá-López F, De LarreaBaz N, Álvarez-Martín E, Morat-Ginistar C. The burden of premature mortality in Spain using standar expected years of life lost: a population-based study. BMC Public Health. 2011; 11(787).

10. Macakobkenbach JP, Kulhánová I, Menvielle G, Bopp $\mathrm{M}$, Borrell C, Costa G, et al. Trends in inequalities in premature mortality: a study of 3.2 million deaths in 13 European countries. J Epidemiol Community Health. 2014 June 25, 2014.

11. Hanly P, Soerjomataram I, Sharp L. Measuring the societal burden of cancer: The cost of lost productivity due to premature cancer-related mortality in Europe. Int J Cancer. 2014 Jul 28.

12. Hanly PA, Sharp L. The cost of lost productivity due to premature cancer-related mortality: an economic measure of the cancer burden. BMC cancer. 2014; 14: 224.

13. Gomez-de la Camara A, Pinilla-Dominguez P, Vazquez-Fernandez Del Pozo S, Garcia-Perez L, Rubio-Herrera MA, Gomez-Gerique JA, et al. Costs resulting from premature mortality due to cardiovascular causes: A 20 year follow-up of the DRECE study. Rev Clin Esp 2014 Jul 4.

14. Akobundu E, Ju J, Blatt L, Mullins CD. Cost-of-illness studies: a review of current methods. Pharmacoeconomics. 2006;24(9):869-90.

15. Luengo-Fernandez R, Leal J, Gray A, Sullivan R. Economic burden of cancer across the European Union: a population-based cost analysis. Lancet Oncol. 2013 Nov;14(12):1165-74.

16. Luengo-Fernandez R, Leal J, Gray AM. Cost of dementia in the pre-enlargement countries of the European Union. J Alzheimers Dis. 2011;27(1):187-96

17. Oliva-Moreno J. Loss of labour productivity caused by disease and health problems: what is the magnitude of its effect on Spain's Economy? Eur J Health Econ. 2012 Oct; 13(5): 605-14.

18. Oliva-Moreno J. Pérdidas de producción laboral ocasionadas por los fallecimientos prematuros en España en el año 2005. Rev Esp Salud Pública. 2009; 83: 123-35.

19. Becker G. The production of human capital. Chicago: Columbia University Press; 1964.

20. Fuchs VR. The contribution of health services to the American economy. Milbank Mem Fund Q Health Soc. 1966; 44: 65-102.

21. Grossman M. On the concept of health capital and the demand for health. J Polit Econ. 1972; 80(2): 223-55.

22. Grossman M. The human capital model of the demand for health. En: Newhouse JP, Culyer AJ. Amsterdam: Handbook of Health Economics; 2000.
23. Encuesta de Estructura Salarial. Instituto Nacional de Estadística. 2005. (citado 14/01/2013). Disponible en: http://www.ine.es/jaxi/menu.do? type=pcaxis\&path=/ $\mathrm{t} 22 / \mathrm{p} 133 \&$ file $=$ inebase $\& \mathrm{~L}=0$.

24. Encuesta de Estructura Salarial. Instituto Nacional de Estadística. 2007. (citado 14/01/2013) Disponible en: http://www.ine.es/jaxi/menu.do?type $=$ pcaxis\&path=/ $\mathrm{t} 22 / \mathrm{p} 133 \&$ file $=$ inebase $\& \mathrm{~L}=0$.

25. Encuesta de Estructura Salarial. Instituto Nacional de Estadística. 2009. Disponible en: http:// www.ine.es $/$ jaxi/menu.do?type $=$ pcaxis $\&$ path $=/ \mathrm{t} 22 /$ p133\&file $=$ inebase $\& \mathrm{~L}=0$. (consultado 14/01/2013)

26. Regitro de defunciones según la causa de la muerte. Instituto Nacional de Estadística. 2005. (citado 14/01/2013)Disponible en: http://www.ine.es/jaxi/menu. do? type $=$ pcaxis \& path $=/ \mathrm{t} 15 / \mathrm{p} 417 \&$ file $=$ inebase $\& \mathrm{~L}=0$.

27. Regitro de defunciones según la causa de la muerte. Instituto Nacional de Estadística. 2007. Disponible en: http://www.ine.es/jaxi/menu.do?type=pcaxis\&path=/ t15/p417\&file $=$ inebase $\& L=0$. (consultado 14/01/2013)

28. Regitro de defunciones según la causa de la muerte. Instituto Nacional de Estadística. 2009. (citado 14/01/2013)Disponible en: http://www.ine.es/jaxi/menu. do? type $=$ pcaxis\&path $=/ \mathrm{t} 15 / \mathrm{p} 417 \&$ file $=$ inebase $\& \mathrm{~L}=0$.

29. Clasificación estadística internacional de enfermedades y problemas relacionados con la salud. Décima revision. Volumen 3. Ginebra: Organizacion Mundial de la Salud; 2008. Disponible en: http://ais.paho.org/classifications/Chapters/pdf/Volume3.pdf “

30. Encuesta de Población Activa. Instituto Nacional de Estadística. 2005. (citado 14/01/2013). Disponible en: http://www.ine.es/inebaseDYN/epa30308/epa_inicio. htm.

31. Encuesta de Población Activa. Instituto Nacional de Estadística. 2009. Disponible en: http://www.ine.es/ inebaseDYN/epa30308/epa_inicio.htm.

32. Encuesta de Población Activa. Instituto Nacional de Estadística. 2007. (citado 14/01/2013). Disponible en: http://www.ine.es/inebaseDYN/epa30308/epa_inicio. htm.

33. Economic Burden of Illness in Canada. Minister of Public Works and Government Services Canada. 2002. (citado 2012) Disponible en: http://publications.gc.ca/ collections/Collection/H21-136-1998E.pdf

34. Fact Book. Fiscal Year 2012. Washington: National Heart, Lung and Blood Institute. National Institutes of Health; 2012.

35. Koopmanschap MA, van Ineveld BM. Towards a new approach for estimating indirect costs of disease. Soc Sci Med. 1992; 34: 1005-10. 
36. Johannesson M, Karlsson G. The friction cost method: A comment. J Health Econ. 1997; 16: 249-55.

37. Koopmanschap MA, Rutten FF, van Ineveld BM, van Roijen L. The friction cost method for measuring indirect costs of disease. J Health Econ. 1995 Jun; 14(2): 171-89.

38. Liljas B. How to calculate indirect costs in economic evaluation. Pharmacoeconomics. 1998; 13: 1-7.

39. Krol M, Brouwer W, Rutten FF. Productivity costs in economic evaluations: past, present, future. Pharmacoeconomics. 2013; 31: 537-40.

40. Nyman JA. Productivity costs revisited: toward a new US policy. Health Economics. 2012; 21(12): 1387401 .

401. Zhang W, Bansback N, Anis AH. Measuring and valuing productivity loss due to poor health: A critical review. Soc Sci Med. 2011; 72: 185-92.

42. MSPSI. Estrategia en Cardiopatía Isquémica del Sistema Nacional de Salud. 2006. (consultado 14/01/2013. Disponible en: http://www.msc.es/organizacion/sns/planCalidadSNS/pdf/excelencia/cancercardiopatia/CARDIOPATIA/opsc_est20.pdf

43. MSPSI. Estrategia en Cáncer del Sistema Nacional de Salud. 2006. (consultado 14/01/2013). Disponible en:http://www.msc.es/organizacion/sns/planCalidadSNS/pdf/excelencia/cancer-cardiopatia/CANCER/ opsc_est1.pdf.pdf

44. MSPSI. Estrategia en Diabetes del Sistema Nacional de Salud. 2007. Disponible en: http://www.msc. es/organizacion/sns/planCalidadSNS/pdf/excelencia/ cuidadospaliativos-diabetes/DIABETES/estrategia_diabetes_sistema_nacional_salud.pdf (consultado $14 / 01 / 2013$ )

45. MSPSI. Estrategia en Ictus del Sistema Nacional de Salud. 2009. (consultado 14/01/2013). Disponible en: http://www.msc.es/organizacion/sns/planCalidadSNS/ docs/EstrategiaIctusSNS.pdf

46. MSPSI. Estrategia de promoción de la salud y prevención en el SNS. (consultado 30/09/2014). Disponible en: http://www.msssi.gob.es/profesionales/ saludPublica/prevPromocion/Estrategia/docs/EstrategiaPromocionSaludyPrevencionSNS.pdf 